\title{
Induced gravitational collapse at extreme cosmological distances: the case of GRB 090423
}

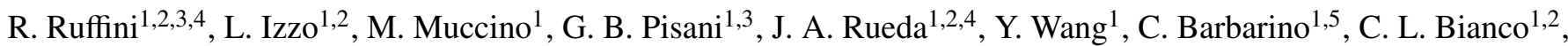 \\ M. Enderli ${ }^{1,3}$, and M. Kovacevic ${ }^{1,3}$ \\ 1 Dip. di Fisica and ICRA, Sapienza Università di Roma, P.le Aldo Moro 5, 00185 Rome, Italy \\ e-mail: Ruffini@icra.it \\ 2 ICRANet, Piazza della Repubblica 10, 65122 Pescara, Italy \\ 3 Université de Nice-Sophia Antipolis, Cedex 2, Grand Château Parc Valrose, Nice, France \\ ${ }^{4}$ ICRANet-Rio, Centro Brasileiro de Pesquisas Fisicas, Rua Dr. Xavier Sigaud 150, 22290-180 Rio de Janeiro, RJ, Brazil \\ 5 INAF-Napoli, Osservatorio Astronomico di Capodimonte, Salita Moiariello 16, 80131 Napoli, Italy
}

Received 17 January 2014 / Accepted 25 May 2014

\begin{abstract}
Context. The induced gravitational collapse (IGC) scenario has been introduced in order to explain the most energetic gamma ray bursts (GRBs), $E_{\text {iso }}=10^{52}-10^{54} \mathrm{erg}$, associated with type $\mathrm{Ib} / \mathrm{c}$ supernovae $(\mathrm{SNe})$. It has led to the concept of binary-driven hypernovae $(\mathrm{BdHNe})$ originating in a tight binary system composed by a FeCO core on the verge of a SN explosion and a companion neutron star (NS). Their evolution is characterized by a rapid sequence of events: 1) the SN explodes, giving birth to a new NS ( $v$ NS). The accretion of SN ejecta onto the companion NS increases its mass up to the critical value; 2) the consequent gravitational collapse is triggered, leading to the formation of a black hole (BH) with GRB emission; 3) a novel feature responsible for the emission in the $\mathrm{GeV}, \mathrm{X}$-ray, and optical energy range occurs and is characterized by specific power-law behavior in their luminosity evolution and total spectrum; 4) the optical observations of the SN then occurs.

Aims. We investigate whether GRB 090423, one of the farthest observed GRB at $z=8.2$, is a member of the BdHN family.

Methods. We compare and contrast the spectra, the luminosity evolution, and the detectability in the observations by Swift of GRB 090423 with the corresponding ones of the best known BdHN case, GRB 090618.

Results. Identification of constant slope power-law behavior in the late X-ray emission of GRB 090423 and its overlapping with the corresponding one in GRB 090618, measured in a common rest frame, represents the main result of this article. This result represents a very significant step on the way to using the scaling law properties, proven in Episode 3 of this BdHN family, as a cosmological standard candle.

Conclusions. Having identified GRB 090423 as a member of the BdHN family, we can conclude that SN events, leading to NS formation, can already occur, namely at $650 \mathrm{Myr}$ after the Big Bang. It is then possible that these BdHNe stem from 40-60 $M_{\odot}$ binaries. They are probing the Population II stars after the completion and possible disappearance of Population III stars.
\end{abstract}

Key words. gamma-ray burst: general - gamma-ray burst: individual: GRB 090423 - black hole physics

\section{Introduction}

The induced gravitational collapse (IGC) paradigm (Ruffini 2011; Rueda \& Ruffini 2012; Izzo et al. 2012b) has been proposed to explain a class of very energetic $\left(E_{\text {iso }} \sim 10^{52}-10^{54} \mathrm{erg}\right)$ long gamma ray bursts (GRBs) associated with supernovae $(\mathrm{SNe})$. A new class of systems, with progenitor a tight binary composed by a FeCO core and a companion neutron star (NS), has been considered. These systems evolve in a very rapid sequence lasting a few hundred seconds in their rest frame: 1) the SN explodes giving birth to a new NS $(v \mathrm{NS}) ; 2)$ the accretion of the SN ejecta onto the companion NS increases its mass, reaching the critical value; 3) the gravitational collapse is triggered, leading to the formation of a black hole (BH) with GRB emission. Such systems have been called binary-driven hypernovae (BdHN Ruffini et al. 2014a).

Observationally, this authentic cosmic matrix is characterized by four distinct episodes, with the "in" state represented by a FeCO core and a NS and the "out" state by a $v \mathrm{NS}$ and a BH. Each episode contains specific signatures in its spectrum and luminosity evolution. Up to now, the IGC paradigm has been verified in a dozen GRBs, all with redshift up to $z \sim 1$ (Izzo et al. 2012a; Penacchioni et al. 2012, 2013; Pisani et al. 2013; Ruffini et al. 2013).

Various approaches have been followed to reach an understanding of long GRBs. One of these has been the use of statistical tools to obtain general results that examine the most complete source catalog (see, e.g., Nousek et al. 2006; Kann et al. 2011; Salvaterra et al. 2012; Margutti et al. 2013, and references therein).

We follow a different approach here. We first identified the specific class of BdHNe of GRBs related to $\mathrm{SNe}$, as mentioned above, widely tested at $z \approx 1$. We furthermore explore the members of this class by extending our analysis to higher values of the cosmological redshifts. We do that by taking the scaling laws for the cosmological transformations into account, as well as the 


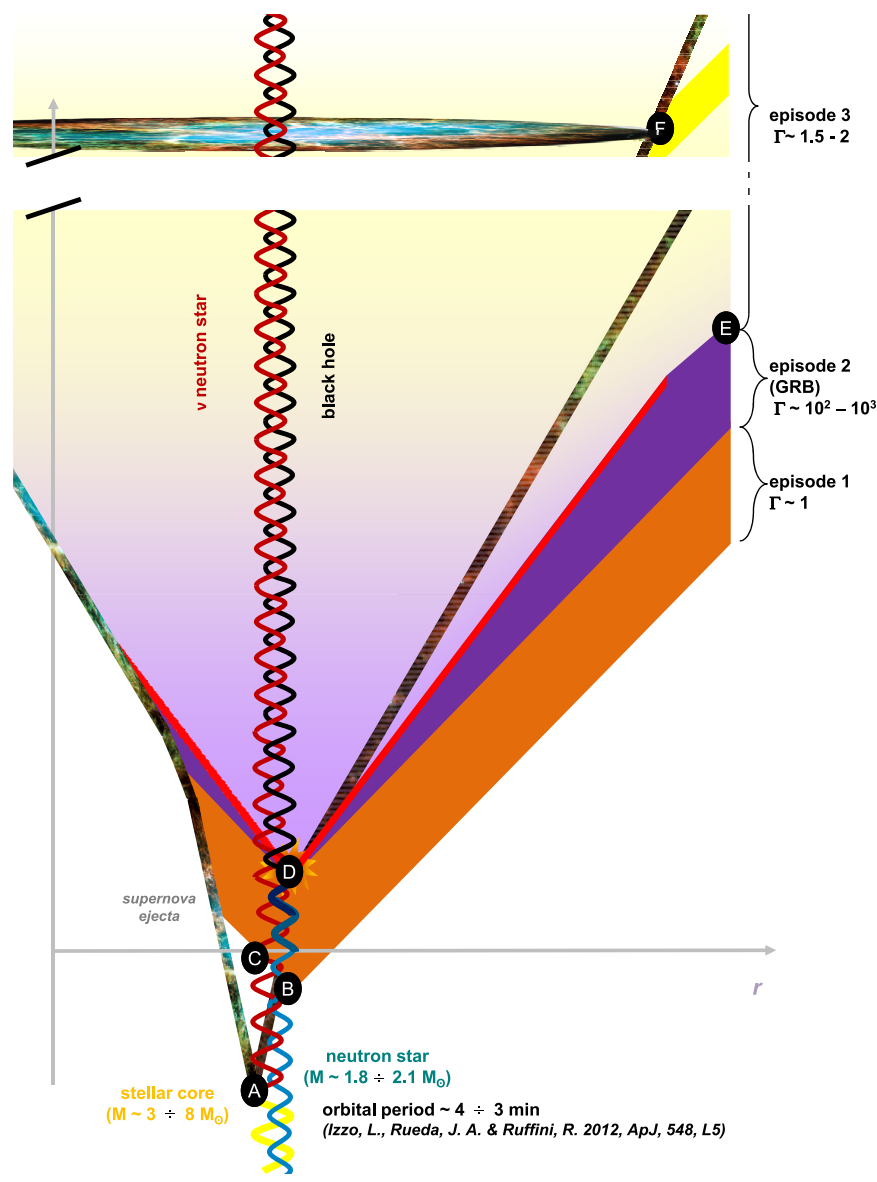

Fig. 1. Space-time diagram of the induced gravitational collapse applied to GRB 090618 (Enderli 2013; Ruffini 2013). The sequence is summarized as follows: A) the explosion as a $\mathrm{SN}$ of the evolved $\mathrm{FeCO}$ core which creates a $v$-NS and its remnant; B) the beginning of the accretion of the SN ejecta onto the companion NS, emitting Episode 1; C) a prolonged interaction between the $v$-NS and the NS binary companion; D) the companion NS reaches its critical mass by accretion, and a $\mathrm{BH}$ is formed with the consequent emission of a GRB; E) the arrival time at the separatrix between Episodes 2 and 3; F) the optical emission of the $\mathrm{SN}$ due to the decay of ${ }^{56} \mathrm{Ni}$ after $t_{\mathrm{a}}^{\mathrm{d}} \sim 10(1+z)$ days in the observer frame (Episode 4).

specific sensitivities of the GRB detectors (in this case Swift, Gehrels et al. 2005; and Fermi, Meegan et al. 2009).

Our aim is to verify that such BdHNe, originating in a $\mathrm{SN}$ and a companion NS, did form in the earliest phases of the universe. If this is confirmed, we go on to examine the possibility that all GRBs with $E_{\text {iso }} \sim 10^{52}-10^{54} \mathrm{erg}$ are indeed associated to $\mathrm{SN}$ and belong to the BdHN family independently of their space and time location.

\section{The four episodes of BdHNe sources}

In order to achieve this goal, we recall the four above-mentioned episodes, present in the most general BdHN (see Fig. 1):

Episode 1 has the imprint of the onset of a SN in the tight binary system with the companion neutron star (NS; see Fig. 2). It stemmed from the hyper-critical accretion of the $\mathrm{SN}$ matter ejecta $\left(\sim 10^{-2} M_{\odot} \mathrm{s}^{-1}\right)$ (Rueda \& Ruffini 2012). Decades of conceptual progress have passed from the original work of Bondi \& Hoyle (1944) and Bondi (1952) to the problem of a "hypercritical" accretion rate. This problem has acquired growing scientific interest as it moved from the classical astronomical field to the

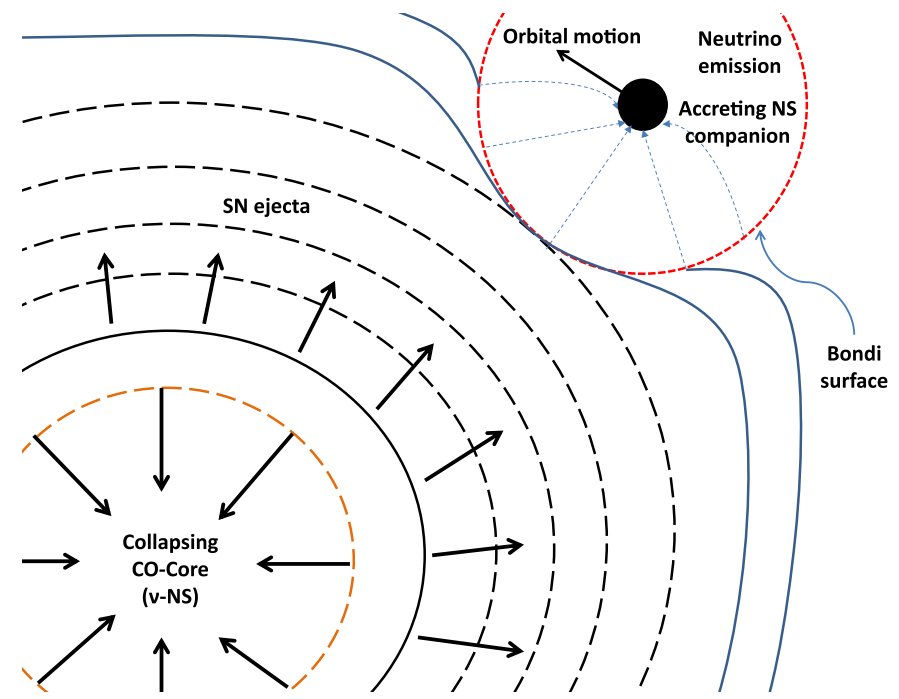

Fig. 2. Sketch (not in scale) of the accretion induced gravitational collapse (IGC) scenario.

domain of the relativistic astrophysics. The crucial role of neutrino cooling, earlier considered by Zel'dovich et al. (1972) and later on by Bisnovatyi-Kogan \& Lamzin (1984) in SN fallback, has been recognized to play a crucial role in describing binary common envelope systems by Chevalier $(1989,1993)$. In the work by Fryer et al. (1996), and more recently in Fryer (2009), it was clearly shown that an accretion rate $\dot{M} \sim 10^{-2} M_{\odot} \mathrm{s}^{-1}$ onto a neutron star (NS) could lead in a few seconds to the formation of a black hole (BH), when neutrino physics in the description of the accreting NS is taken into due account. The data acquired in Episode 1 of GRB 090618 (Izzo et al. 2012a), as well as the one in GRB 101023 (Penacchioni et al. 2012), GRB 110709B (Penacchioni et al. 2013), and GRB 970828 (Ruffini et al. 2013), give for the first time the possibility to probe the Bondi-Hoyle hypercritical accretion and possibly the associated neutrino emission, which was theoretically considered by Zel'dovich et al. (1972); Chevalier (1993); Fryer et al. (1996), and Fryer (2009).

Episode 2 is the canonical GRB emission, which originated in the collapse of the companion NS, which reached its critical mass by accretion of the SN ejecta and then collapsed to a black hole $(\mathrm{BH})$, indeed emitting the GRB.

Episode 3 observed in X-rays by Swift-XRT, shows very precise behavior consisting of steep decay, starting at the end point of the prompt emission, and then a plateau phase followed by a late power-law decay (see Pisani et al. 2013 and also Fig. 3). The late X-ray luminosities of BdHNe, in their rest-frame energy band $0.3-10 \mathrm{keV}$, show a common power-law behavior with a constant decay index clustering around $\alpha=-1.5 \pm 0.2$. The occurrence of such a constant afterglow decay has been observed in all the BdHN sources examined. For example, see in Fig. 4 the data for GRB 130427A, GRB 061121, GRB 060729, respectively. It appears an authentic nested structure, in the late X-ray emission of GRBs associated to $\mathrm{SNe}$, and it has indeed to be indicated as the qualifying feature for a GRB to be a member of the BdHNe family (Ruffini et al. 2014a). It is clear that such a phenomenon offers a strong challenge for explaining by any GRB model.

In addition to these X-ray features, the observations of GRB 130427A by the Swift, Fermi, and Konus-WIND satellites and a large number of optical telescopes have led to the evidence of such power laws at very high energies, in $\gamma$-rays and 


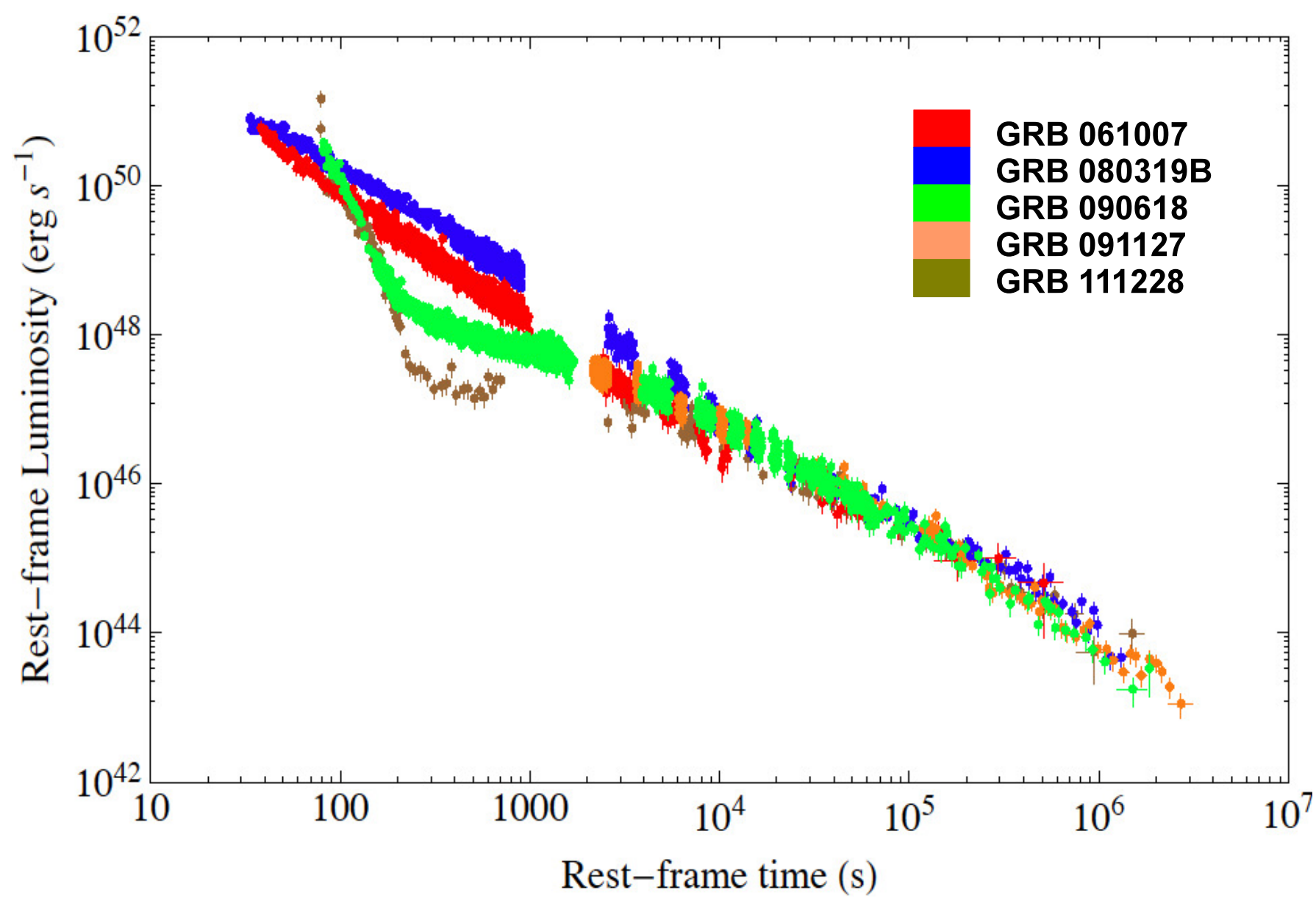

Fig. 3. Rest-frame, X-ray afterglow, luminosity light curves of some IGC GRBs-SNe belonging to the "golden sample" described in Pisani et al. (2013). The overlapping after $10^{4} \mathrm{~s}$ is clearly evident, confirming the presence of an Episode 3 in this GRB.

at the optical wavelengths (Fermi-LAT collaboration \& FermiGBM collaboration 2014; Melandri et al. 2014; see also Ruffini et al. 2014b).

Episode 4 is characterized by the emergence of the SN emission after about 10-15 days from the occurrence of the GRB in the rest frame of the source, which has been observed for almost all the sources fulfilling the IGC paradigm with $z \sim 1$.

\section{GRB 090423 compared and contrasted with GRB 090618}

We first consider the data of GRB 090423, one of the farthest GRB ever observed at $z=8.2$ (Salvaterra et al. 2009; Tanvir et al. 2009), with the prototypical member of the BdHNe class, namely GRB 090618, and its associated SN (Izzo et al. 2012a). In other words we proceed with a specific ansatz: we verify that GRB 090423, at $z=8.2$, presents analogous intrinsic features to GRB 090618, which was observed at $z=0.54$.

We proceed by examining (see Sect. 4) each one of the above episodes for both sources, by a detailed spectral analysis and simulations. We first verify that Episode 1 of GRB 090618 transposed at redshift $z=8.2$ should not have triggered the Swift-BAT detector. Indeed, no precursor in the light curve of GRB 090423 was detected. Consequently, we do not address any theoretical considerations of the hypercritical accretion in Episode 1 of GRB 090423, since it is not observable in this source (see Sect. 5). We also notice that the distance of
GRB 090423 prevents any possible detection of a SN associated with this GRB, and therefore Episode 4 cannot be observed in GRB 090423.

For Episode 2, we have found that indeed the transposed emission of GRB 090618 should provide a positive trigger: we show in Sect. 6 that the duration, the observed luminosity and the spectral emission of Episode 2 in GRB 090423 present analogous intrinsic features to the transposed ones of GRB 090618 and differ only in the spectral energy distribution due to different circumburst medium properties.

For Episode 3, the crucial result, probing the validity of the above ansatz, is that the late X-ray emission in GRB 090423, computed in the rest frame of the burst at $z=8.2$, precisely coincides (overlaps) with the corresponding late X-ray emission in GRB 090618, as evaluated in the rest frame of the source at $z=0.54$, see Sect. 7 . The occurrence of this extraordinary coincidence in Episode 3 proves that GRB 090423 is indeed a member of the BdHN family. This in particular opens the possibility of elaborating a role for the late X-ray emission in BdHNe as a standard candle.

\section{The data}

GRB 090423 was discovered on 23 April 2009, 07:55:19 UT, $T_{0}$ from here, by the Swift Burst Alert Telescope (BAT; Krimm et al. 2009), at coordinates RA $=09^{\mathrm{h}} 55^{\mathrm{m}} 35^{\mathrm{s}}$, Dec $=$ $+18^{\circ} 09^{\prime} 37^{\prime \prime}$ (J2000.0; $3^{\prime}$ at $90 \%$ containment radius). The 

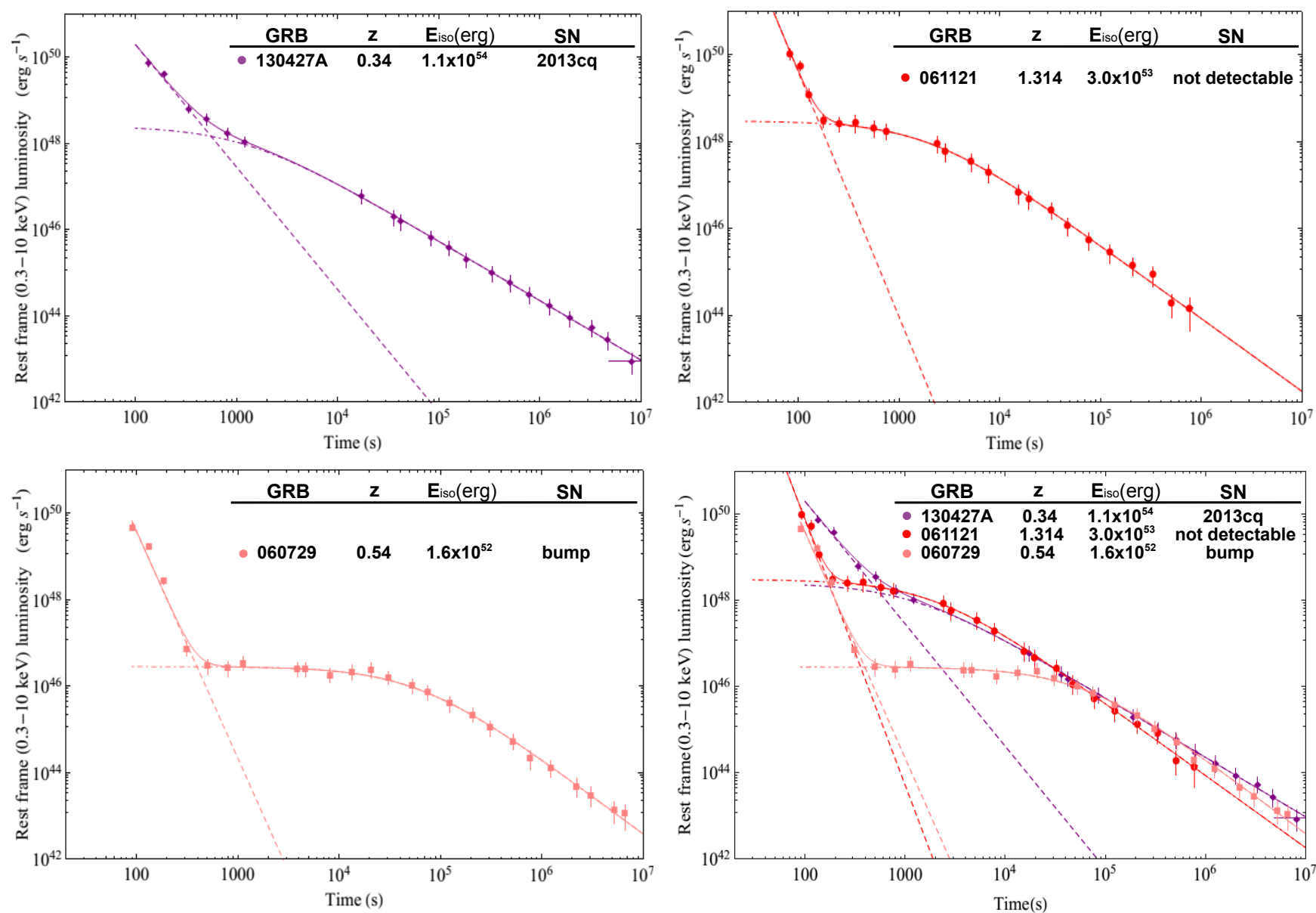

Fig. 4. Rest-frame, (0.3-10) keV, and re-binned luminosity light curves of GRB 130427A (upper left), GRB 061121 (upper right), GRB 060729 (lower left) and a combined picture (lower right). The fits to their emission is done using a power-law function for the early steep decay and a phenomenological function for the following emission, which is described well in Ruffini et al. (2014a).

Swift-BAT light curve showed a double-peaked structure with a duration of about $20 \mathrm{~s}$. The X-ray Telescope (XRT; Burrows et al. 2005) on board the same spacecraft started to observe GRB $09042372.5 \mathrm{~s}$ after the initial trigger, finding a fading source and providing enhanced coordinates for the follow-up by on-ground telescopes that have allowed the discovery of its redshift $(z=8.2$, Salvaterra et al. 2009; Tanvir et al. 2009). The light curve is characterized by an intense and long flare peaking at about $T_{0}+180$, followed by a power-law decay, observed from the second orbit of Swift (Stratta \& Perri 2009). The prompt emission from GRB 090423 was also detected by the Fermi Gamma-Ray Burst Monitor (GBM, trigger 262166127/090423330; von Kienlin 2009a), whose onground location was consistent with the Swift position. The Large Area Telescope (LAT) on-board the Fermi satellite did not detected any signal from GRB 090423. The GBM light curve showed a single-structured peak with a duration of about $12 \mathrm{~s}$, whose spectral energy distribution was best fit with a power law with an exponential cut-off energy, parameterized as $E_{\text {peak }}=(82 \pm 15) \mathrm{keV}$. The observed fluence was computed from Fermi data to be $S_{\gamma}=1.1 \times 10^{-6} \mathrm{ergs} / \mathrm{cm}^{2}$ that, considering the standard $\Lambda \mathrm{CDM}$ cosmological model, corresponds to an isotropic energy emitted of $E_{\text {iso }}=1.1 \times 10^{53} \mathrm{ergs}$ for the spectroscopic redshift $z=8.2$ (von Kienlin 2009b). With these values for $E_{\text {peak }}$ and $E_{\text {iso }}$, GRB 090423 satisfies the Amati relation, which is only valid for long GRBs (Amati et al. 2002).

\section{The impossibility of detecting Episode 1}

It has become natural to ask if observations of Episodes 1 and 2 in the hard X-ray energy range could be addressed for the case of GRB 090423. We have first analyzed a possible signature of Episode 1 in GRB 090423. Since the Swift-BAT, (15-150) keV, light curve is a single-structured peak with duration of $\sim 19 \mathrm{~s}$, as detected by Swift-BAT, with no thermal emission in its spectrum and no detection of any emission from a precursor in the Swift and Fermi data, we have considered the definite possibility that Episode 1 was not observed at all. In this light, the best way to check this possibility consists in verifying that the Episode 1 emission is below the threshold of the Swift-BAT detector, consequently, it could have not triggered the Swift-BAT. We have considered the prototype of Episode 1 as the one observed in GRB 090618 (Izzo et al. 2012b), which is at redshift $z=0.54$, and then we transposed it at redshift $z=8.2$, simulating the observed emission of GRB 090618 as if it had been observed at this large distance. Then, we performed a time-resolved spectral analysis of Episode 1 in GRB 090618, using a Band function as spectral model, and finally we translated the specific photon spectra obtained from the analysis at the redshift of GRB 090423. This last operation consists in two transformations, concerning the peak energy $E_{\text {peak }}$ of the Band function and the normalization value $K_{\text {Band }}$. The new value of the peak energy is simply given by $E_{\text {peak }, 8}=E_{\text {peak }}(1+0.54) /(1+8.2)$, while the 
normalization, which corresponds to the specific photon flux at $1 \mathrm{keV}$, requires knowledge of the luminosity distances of the two bursts, $d_{l}(z)$ :

$K_{\text {Band }, 8}=K_{\text {Band }}\left(\frac{1+8.2}{1+0.54}\right)^{2}\left(\frac{d_{l}(0.54)}{d_{l}(8.2)}\right)^{2}$.

Another transformation concerns the observational time of Episode 1 of GRB 090618 at redshift $z=8.2$. At large distances, any astrophysical event will be dilated in time by the cosmological redshift effect, which in the current case modifies the time interval by a quantity $(1+8.2) /(1+0.54)=5.97$. The knowledge of this time interval is fundamental since it represents the exposure of a simulated spectrum translated at $z=8.2$. We considered Fermi GBM data for analyzing the time-resolved spectra of GRB 090618, as described by Izzo et al. (2012b). The wide energy range of Fermi GBM NaI detectors, (8-1000) keV, allows a more accurate determination of the Band parameters, which are used as input values for the simulated spectra. We also rebinned the Fermi data considering a signal-to-noise ratio $(\mathrm{SNR})=10$, and finally performed our spectral analysis. The next step consisted in transforming the peak energy of the Band function and of the normalization of all these time-resolved photon spectra $N(E)$, as described above.

Following the work of Band (2003), the sensitivity of an instrument to detect a burst depends on its burst trigger algorithm. The Swift-BAT trigger algorithm, in particular, looks for excesses in the detector count rate above expected background and constant sources. There are several criteria for determining the correct BAT threshold significance $\sigma_{0}$ for a single GRB (Barthelmy et al. 2005), but in this work we have considered the treatment given in Band (2003). Recently, the threshold of Swift-BAT has been modified to allow detecting of subthreshold events, but since GRB 090423 was detected before, the Band (2003) analysis is still valid for our purposes. The preset threshold significance for Swift-BAT can be expressed by the following formula:

$\sigma_{0}=\frac{A_{\mathrm{eff}} f_{\mathrm{det}} f_{\mathrm{mask}} \Delta t \int_{15}^{150} \epsilon(E) N(E) \mathrm{d} E}{\sqrt{A_{\mathrm{eff}} f_{\mathrm{det}} \Delta t \int_{15}^{150} B(E) \mathrm{d} E}}$,

where $A_{\text {eff }}$ is the effective area of the detector, $f_{\text {det }}$ the fraction of the detector plane that is active, $f_{\text {mask }}$ the fraction of the coded mask that is open, $\Delta t$ the exposure of the photon spectrum $N(E)$, $\epsilon(E)$ the efficiency of the detector, and $B(E)$ the background. We considered the values for these parameters as the ones given in the Band work (with the exception of the detecting area, assumed to be $A_{\text {eff }}=5200 \mathrm{~cm}^{2}$ ), while the efficiency and the background were obtained from the Swift-BAT integrated spectrum of GRB 090423 using the XSPEC fitting package. Then we considered as input photon spectra $N(E)$ the ones obtained from the Fermi GBM analysis of Episode 1 of GRB 090618 and translated for the redshift $z=8.2$. It is appropriate to note that the transformations of spectra presented above are the correct ones: since the sensitivity of Swift-BAT strongly depends on the peak energy of the photon flux of the single spectra of the GRB (for the Swift-BAT case, see e.g. Fig. 7 of Band 2003), we find that at $z=8.2$ the observed peak energies of any spectrum will be lowered by a factor $(1+0.54) /(1+8.2)$. Our procedure also takes this further effect of the cosmological redshift into account.

Since the threshold significance of Swift-BAT is variable from a minimum value of $\sigma_{0}=5.5$ up to a maximum value of $11^{1}$, with an average value of $\sigma_{0}=6.7$, the results of this

\footnotetext{
1 http://swift.gsfc.nasa.gov/about_swift/bat_desc.html
}

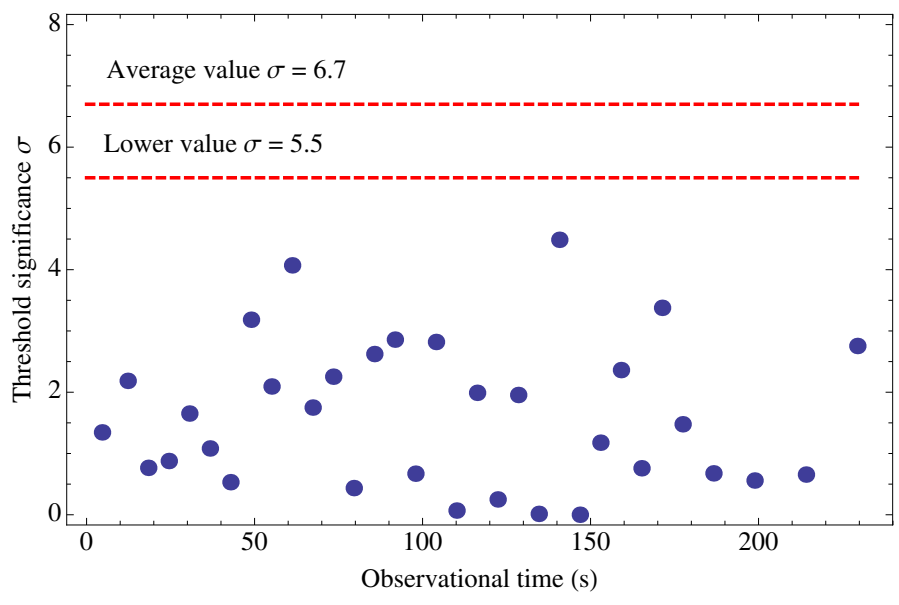

Fig. 5. Threshold significance $\sigma_{0}$ computed using the treatment of Band (2003) for any single time-resolved spectra of the first emission episode in GRB 090618, as if they were emitted at redshift 8.2. The dashed lines correspond to the values for the threshold significance of $\sigma_{0}=5.5$ and $\sigma_{0}=6.7$.

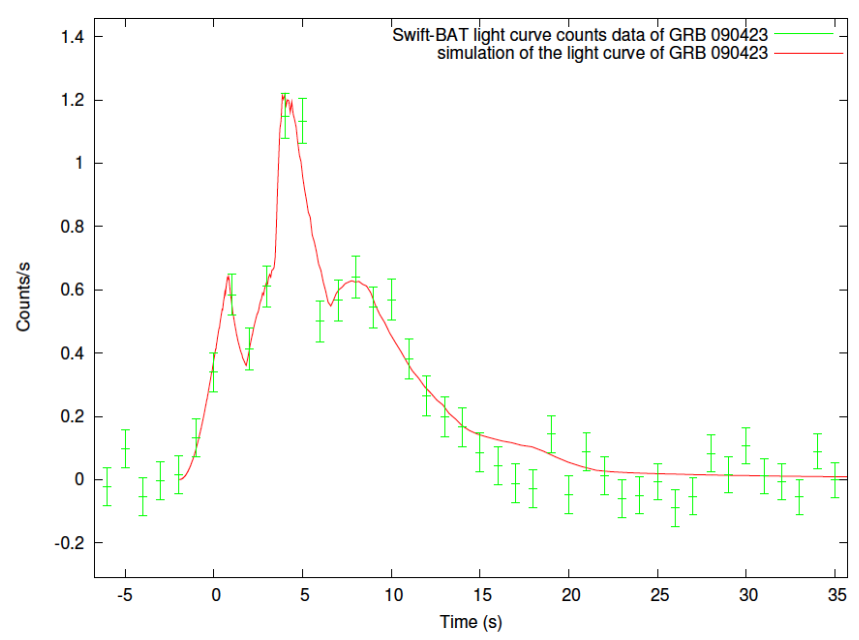

Fig. 6. Swift-BAT (15-150 keV) light curve emission of GRB 090423. The red line corresponds to the simulation of the GRB emission in the fireshell scenario (Izzo et al. 2010).

first analysis suggest that an Episode 1 similar to the one of GRB 090618 would not have been detected in GRB 090423 (see Fig. 5).

\section{Detection of Episode 2 and its analysis}

Episode 2 emission of GRB 090423, detected by Swift-BAT, was examined in the context of the fireshell scenario (Izzo et al. 2010; Ruffini 2011). A Lorentz Gamma factor of $\Gamma \sim 1100$ and a baryon load $B=8 \times 10^{-4}$ were obtained. The simulations of the observed spikes in the observed time interval $(0-440) \mathrm{s}$ lead to homogeneous circumburst medium $\left(\mathcal{R}=10^{-8}\right.$, see Bianco \& Ruffini 2005 for a complete description), and an average density of $10^{-1}$ particles $\mathrm{cm}^{-3}$. The simulation of the GRB 090423 emission is shown in Fig. 6.

We can now compare and contrast the emission observed in GRB 090423, expressed at $z=8.2$ (see Fig. 6, Izzo et al. 2010), and the portion of the emission of GRB 090618 if observed at $z=8.2$, (see Fig. 7, Izzo et al. 2012a). In view of the Swift-BAT threshold, only the dashed region in Fig. 8, lasting 6 s, would be 
Table 1. Results of the spectral fits of the T90 duration of GRB 090423 and of the $\Delta t_{A, \text { obs }}$ time interval for GRB 090618 .

\begin{tabular}{ccccccc}
\hline \hline & $\alpha$ & $\begin{array}{c}\beta \\
(\mathrm{keV})\end{array}$ & $\begin{array}{c}E_{\mathrm{p}, \mathrm{i}} \\
(\mathrm{keV})\end{array}$ & $\begin{array}{c}\text { norm. } \\
\left(\mathrm{ph} / \mathrm{cm}^{2} / \mathrm{s} / \mathrm{keV}\right)\end{array}$ & $\tilde{\chi}^{2}$ & $\begin{array}{c}\Delta t_{\mathrm{obs}} \\
(\mathrm{s})\end{array}$ \\
\hline 090618 & $-0.66 \pm 0.57$ & $-1.99 \pm 0.05$ & $284.57 \pm 172.10$ & $0.3566 \pm 0.16$ & 0.924 & 6.1 \\
090423 & $-0.78 \pm 0.34$ & $-3.5 \pm 0.5$ & $433.6 \pm 133.5$ & $0.015 \pm 0.010$ & 0.856 & 10.4 \\
\hline
\end{tabular}

Notes. The latter is computed in a time interval corresponding to the one expected to be observed if GRB 090618 is transposed at the redshift $z=$ 8.2 , and in the observed energy range (89.6-896) keV.

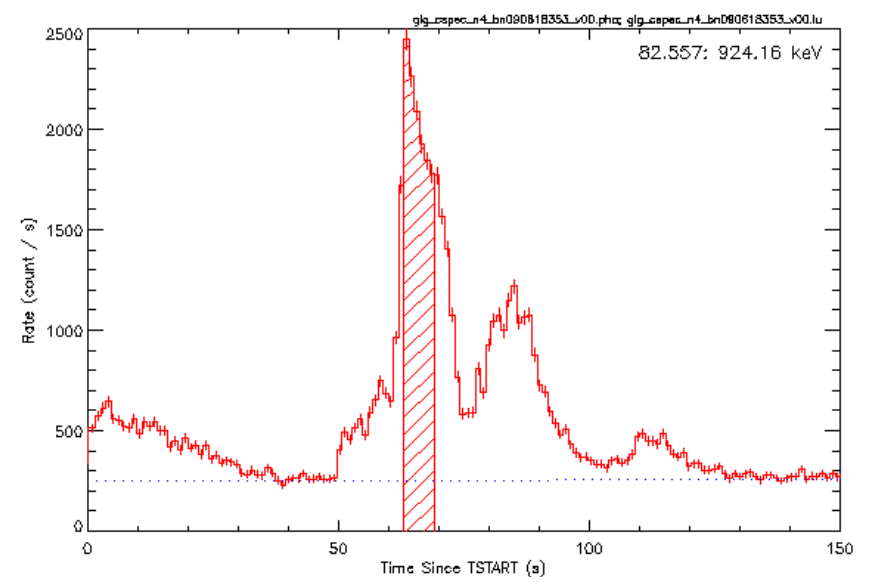

Fig. 7. Light curve of Episode 2 in GRB 090618, ranging from 50 to $150 \mathrm{~s}$. The dashed region represents the portion which would have triggered the Swift-BAT if this GRB had been at the redshift $z=8.2$. The observed duration of that interval is approximately $\Delta t \simeq 6 \mathrm{~s}$. The results obtained in Fig. 6, when scaled to $z=0.54$, provide $\Delta T \simeq 3 \mathrm{~s}$.

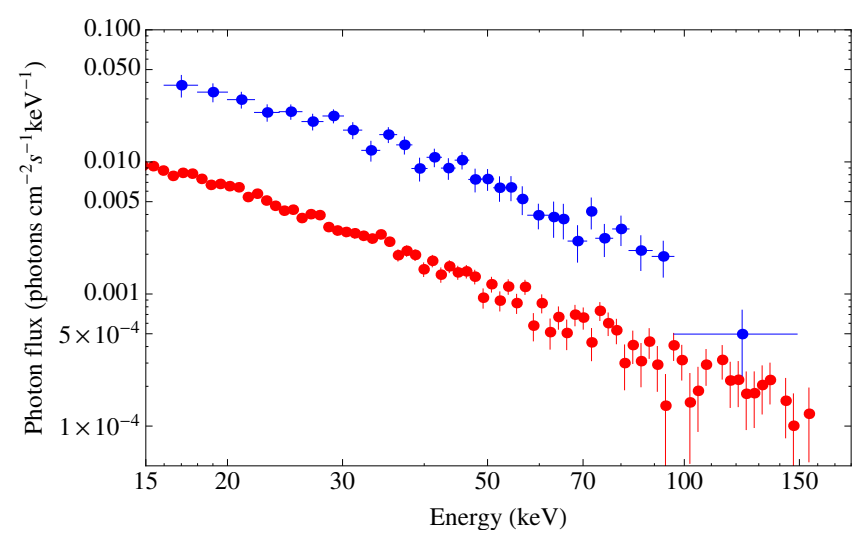

Fig. 8. Spectra of GRB 090423 (blue data) and of the spectrum of the emission of GRB 090618 (red data) considered as possible Episode 2 if GRB 090618 had been observed at $z=8.2$. The low-energy photon index is $\approx-0.8$, which corresponds to the expectations from the Fireshell scenario (Ruffini 2011; Patricelli et al. 2012).

detectable. The observed flux in Fig. 6 and the one of the dashed region in Fig. 8 will be similar when compared in a common frame.

For the above considerations, the analysis presented in the previous section can be applied to Episode 2 of GRB 090618. Assuming a detector threshold for Swift-BAT of $\sigma_{0}=6.7$, see Eq. (2), only the dashed region in Fig. 7 is detectable when transposing GRB 090618 at $z=8.2$. In the observer frame, this emission corresponds to the time interval $\left(T_{0, \mathrm{G}}+63.0\right.$, $\left.T_{0, \mathrm{G}}+69.1\right) \mathrm{s}$, with $T_{0, \mathrm{G}}$ the trigger time of Fermi GBM data of GRB 090618. This time interval, at $z=8.2$, has a duration $\Delta t_{A, \mathrm{obs}}=\Delta t_{\mathrm{obs}} \times 5.97=36.4 \mathrm{~s}$, owing to the time dilation by the cosmological redshift $z$ (see Fig. 6). The remaining emission of GRB 090618 is unobservable, since below the threshold of the Swift-BAT detector. We note that $\Delta t_{A, \text { obs }}$ is quite comparable to the observed duration of GRB 090423 (see Fig. 6).

We turn now to comparing and contrasting the spectral energy distributions in the rest frame of the two GRBs. We consider the spectrum of GRB 090618 in the energy range (89.6-896) $\mathrm{keV}$, which corresponds to the Swift-BAT band $(15-150) \mathrm{keV}$ in the rest frame of GRB 090423. As for the time interval in GRB 090423, we consider the observational time interval (63.0-69.1) s, determined from applying Eq. (2) to the entire Episode 2 of GRB 090618 (see the dashed region in Fig. 7). We fitted the spectral emission observed in GRB 090423 with a Band function (Band et al. 1993), and the results provide an intrinsic peak energy $E_{\mathrm{p}, \mathrm{i}}=(284.57 \pm 172.10) \mathrm{keV}$ (see Table 1). The same model provides for the spectral emission of GRB 090423, in the $T_{90}$ time duration, an intrinsic peak energy of $E_{\mathrm{p}, \mathrm{i}}=(433.6 \pm 133.5) \mathrm{keV}$. However, the break in GRB 090423 is steeper, while in GRB 090618 it is more shallow. This is clear in Fig. 8, where we show the spectra of both GRBs that are transformed to a common frame, which is the one at redshift $z=8.2$. Very likely, the difference in the steepening at high energies is related to the structure of the circumburst medium (CBM): the more fragmented the CBM, the larger the cutoff energy of the fireshell spectrum (Bianco \& Ruffini 2005). Another important result is that the low energy index $\alpha$ is quite similar in both GRBs. This agrees with the expectation from the fireshell scenario, where a photon index of $\approx-0.8$ is expected in the early emission of a GRB (Patricelli et al. 2012).

The isotropic energy emitted in the time interval delineated by the dashed region in Fig. 7 has been computed to be $E_{\text {iso }}=$ $3.49 \times 10^{52} \mathrm{erg}$, which is very similar to the one computed for the $T_{90}$ duration, in the same energy range, for GRB 090423, $E_{\text {iso }}=4.99 \times 10^{52} \mathrm{erg}$.

\section{Striking observations of Episode 3}

That in long GRBs the X-ray emission, observed by SwiftXRT in energy range $0.3-10 \mathrm{keV}$, presents a typical structure composed of a steep decay, a plateau phase and a late powerlaw decay, was clearly expressed by Nousek, Zhang and their collaborators (Nousek et al. 2006; Zhang et al. 2006). This structure acquires a special meaning when examined in the most energetic sources, $E_{\text {iso }}=10^{52}-10^{54} \mathrm{erg}$, and leads to the fundamental proof that GRB 090423 is a BdHN source.

It has only been after applying the IGC paradigm to the most energetic long GRBs associated to SNe that we noticed the most unique characterizing property of the BdHN sources: while the steep decay and the plateau phase can be very different from source to source, the late X-ray power-law component overlaps, 


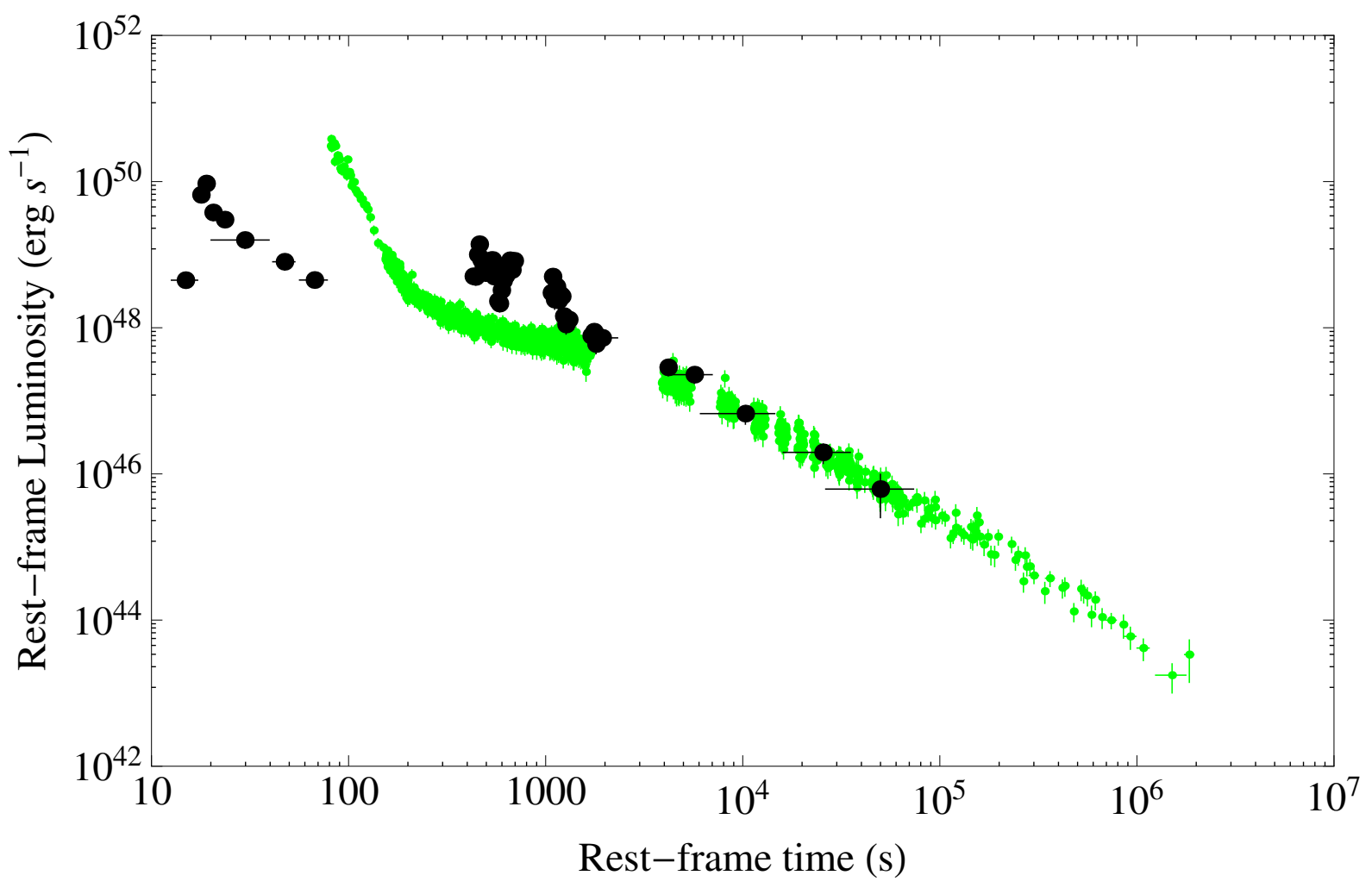

Fig. 9. Behavior of the Episode 3 luminosity of GRB 090423 (black dots) compared with the prototype case of GRB 090618 (green data).

when computed in the cosmological rest-frame (see Pisani et al. 2013 and Fig. 3). This has become the crucial criterion for asserting membership of a GRB in the BdHN family. Indeed, when we report the late X-ray emission of Episode 3 in GRB 090423 at $z=8.2$, and GRB 090618 at $z=0.54$, we observe a complete overlapping at times longer than $10^{4} \mathrm{~s}$, see Fig. 9.

\subsection{Recent progress in understanding the nature of Episode 3}

We recall:

a) that the X-ray luminosity of Episode 3 in all BdHN sources presents precise scaling laws (see, e.g., Fig. 3);

b) that the very high energy emission all the way, up to $100 \mathrm{GeV}$, in GRB 130427A, as well as the optical one, follows a power-law behavior similar to the one in the X-ray emission described above. The corresponding spectral energy distribution is also described by a power-law function (Kouveliotou et al. 2013; Ruffini et al. 2014b). These results clearly require a common origin for this emission process in Episode 3;

c) that an X-ray thermal component has been observed in the early phases of Episode 3 of GRB 060202, 060218, 060418, 060729, 061007, 061121, 081007, 090424,100316D, 100418A, 100621A, 101219B, and 120422A (Page et al. 2011; Starling et al. 2012; Friis \& Watson 2013). In particular, this feature has been clearly observed in GRB 090618 and GRB 130427A (Ruffini et al. 2014b). This implies an emission region size of $10^{12-13} \mathrm{~cm}$ in these early phases of Episode 3, with an expansion velocity of $0.1<v / c<0.9$, with a bulk Lorentz $\Gamma$ factor $\lesssim 2$ (Ruffini et al. 2014a).

The simultaneous occurrence of these three features imposes very stringent constraints on any possible theoretical models. In particular, the traditional synchrotron ultra-relativistic scenario of the Collapsar jet model (Woosley 1993; Meszaros \& Rees 2000) does not appear suitable for explaining these observational facts.

In Ruffini et al. (2014a), we have recently pointed out the possibility of using the nuclear decay of ultra-heavy nuclei originally produced in the close binary phase of Episode 1 by r-process as an energy source of Episode 3. There is the remarkable coincidence that this set of processes leads to the value of the power-law emission with decay index $\alpha$, similar to the one observed and reported in Metzger et al. (2010). The total energy emitted in the decay of these ultra-heavy elements agrees with the observations in Episode 3 of BdHN sources (Ruffini et al. 2014a). An additional possibility of process-generating a scaleinvariant power law in the luminosity evolution and spectrum are the ones expected from type-I and type-II Fermi acceleration mechanisms (Fermi 1949). The application of these acceleration mechanisms to the BdHN remnant has two clear advantages: 1) for us, to fulfill the above-mentioned power laws, both for the luminosity and the spectrum; and 2) for Fermi, to solve the longstanding problem, formulated by Fermi in his classic paper, of identifying the injection source to make his acceleration mechanism operational on an astrophysical level.

\section{Conclusions}

The ansatz that GRB 090423 is the transposed of GRB 090618 at $z=8.2$ has passed scrutiny. It is viable with respect to Episodes 1 and 4 and has obtained important positive results from the analysis of Episodes 2 and 3:

- Episodes 1 and 4 have not been detected in GRB 090423. This is consistent with the fact that the flux of Episodes 1 and 4 of GRB 090618 should not be observed by the 
Swift-BAT detector or by the optical telescopes, owing to the very high redshift of the source and the current sensitivities of X-ray and optical detectors;

- Episode 2 of GRB 090423 has definitely been observed by Swift-BAT: its observed emission is comparable 1) to energy emitted $\left(3.49 \times 10^{52} \mathrm{erg}\right.$ for GRB 090618 and $4.99 \times 10^{52} \mathrm{erg}$ for GRB 090423); 2) to the observed time duration (34 s for the observable part of GRB 090618 when transposed to $z=8.2$ and $19 \mathrm{~s}$ for GRB 090423); and 3) to the spectral energy distribution: the low energy part of the spectra of both GRBs is consistent with the expectation of the fireshell model (Patricelli et al. 2012). There is a significant difference only in the high energy part of the spectrum of GRB 090423, where a cutoff is observed at lower energy than the one in GRB 090618. This can be explained, in the fireshell scenario, by the existence of a dense and homogeneous CBM (Bianco \& Ruffini 2005), which is expected for bursts at high redshifts;

- Episode 3 shows the striking feature of the overlapping of the late X-ray luminosities of Episode 3 in GRB 090618 and GRB 090423, when compared in their cosmological rest frames (see Fig. 9). This result confirms the extension of the relation presented in Pisani et al. (2013) for $z \leq 1$, all the way up to $z=8.2$.

From an astrophysical point of view, all the above results clearly indicate that

a) GRB 090423 is fully consistent with being a member of the BdHN family, and the associated SN did occur already at $z=8.2$ : the possibility of having an evolved binary system about $650 \mathrm{Myr}$ after the Big Bang is not surprising, since the lifetime of massive stars with a mass up to $30 M_{\odot}$ is $\sim 10 \mathrm{Myr}$ (Woosley et al. 2002), which is similar to expectations from normal Population II binary stars also at $z=8.2$, as pointed out by Belczynski et al. (2010);

b) the FeCO core and the NS companion occurring at $z=8.2$ also implies the existence, as the progenitor, of a massive binary $\sim 40-60 M_{\odot}{ }^{2}$. Such massive binaries have recently been identified in $\eta$ Carinae (Damineli et al. 2000). The very rapid evolution of such very massive stars will lead first to a binary X-ray source, like Cen-X3 (see, e.g., Gursky \& Ruffini 1975) and Giacconi \& Ruffini (1978), which will further evolve in the FeCO with the binary NS companion. A similar evolution starting from a progenitor of two very massive stars was considered by Fryer et al. (1999) and by Bethe \& Brown (1998), leading to the formation of binary NSs or postulating the occurrence of GRBs. They significantly differ from the IGC model and also differ in their final outcomes;

c) the results presented in this article open the way to considering the late X-ray power-law behavior in Episode 3 as a distance indicator and represents a significant step toward formulating a cosmological standard candle based on Episode 3 of these BdHN sources.

We turn now to fundamental issues in physics.

1) The traditional fireball jet model (Meszaros 2006) describes GRBs as a single phenomenon, originating in a collapsar (Woosley 1993) and characterized by jet emission moving at Lorentz $\Gamma$ factor in the range $\approx 200-2000$. This contrasts with the BdHN model where the GRB is actually composed

\footnotetext{
2 http://nsm.utdallas. edu/texas2013/proceedings/3/1/ Ruffini.pdf
}

of three different episodes that are conceptually very different among each other (see Fig. 1): Episode 1 is nonrelativistic, and Episode 2 is ultra-relativistic with Lorentz $\Gamma$ factor $\approx 200-2000$, Episode 3 is mildly relativistic, with $\Gamma \approx 2$.

2) The description of Episode 1, see Fig. 2, proposes the crucial role of the Bondi-Hoyle hypercritical accretion process of the SN ejecta onto the NS companion. This requires an urgent analysis of the neutrino emission pioneered in the classic papers of Zel'dovich et al. (1972); Chevalier (1993); Fryer et al. (1996), and (Fryer 2009).

3) The binary nature of the progenitors in the BdHN model and the presence of the specific scaling power laws in the luminosity in Episode 3 of GRB 090423, as well as in all the other sources of the "golden sample" (see Fig. 3; Pisani et al. 2013), has led us to consider the decay of heavy nuclear material originating in $r$-processes (Ruffini et al. 2014a), as well as type-I and type-II Fermi acceleration mechanism as possible energy sources of the mildly relativistic Episode 3 (Ruffini et al. 2014b).

Acknowledgements. We are very grateful to the referee for the very thoughtful considerations and advices which greatly improved the presentation of our manuscript. We thank David W. Arnett for fruitful discussions and support. We are also grateful to Elena Zaninoni and Gennaro De Tommaso for their comments on the manuscript. This work made use of data supplied by the UK Swift Science Data Centre at the University of Leicester. G.B.P., M.E., and M.K. are supported by the Erasmus Mundus Joint Doctorate Program by grant Nos. 2011-1640, 2012-1710, and 2013-1471, respectively, from the EACEA of the European Commission.

\section{References}

Amati, L., Frontera, F., Tavani, M., et al. 2002, A\&A, 390, 81

Band, D. L. 2003, ApJ, 588, 945

Band, D., Matteson, J., Ford, L., et al. 1993, ApJ, 413, 281

Barthelmy, S. D., Barbier, L. M., Cummings, J. R., et al. 2005, Space Sci. Rev., 120,143

Belczynski, K., Holz, D. E., Fryer, C. L., et al. 2010, ApJ, 708, 117

Bethe, H. A., \& Brown, G. E. 1998, ApJ, 506, 780

Bianco, C. L., \& Ruffini, R. 2005, ApJ, 620, L23

Bisnovatyi-Kogan, G. S., \& Lamzin, S. A. 1984, Sov. Astron., 28, 187

Bondi, H. 1952, MNRAS, 112, 195

Bondi, H., \& Hoyle, F. 1944, MNRAS, 104, 273

Burrows, D., Hill, J., Nousek, J., et al. 2005, Space Sci. Rev., 120, 165

Chevalier, R. A. 1989, ApJ, 346, 847

Chevalier, R. A. 1993, ApJ, 411, L33

Damineli, A., Kaufer, A., Wolf, B., et al. 2000, ApJ, 528, L101

Enderli, M. 2013, 27th Texas Symp. on Relativistic Astrophysics, Dallas, TX (USA), December 8-13

Fermi, E. 1949, Phys. Rev., 75, 1169

Fermi-LAT collaboration, \& Fermi-GBM collaboration. 2014, Science, 343, 42

Friis, M., \& Watson, D. 2013, ApJ, 771, 15

Fryer, C. L. 2009, ApJ, 699, 409

Fryer, C. L., Benz, W., \& Herant, M. 1996, ApJ, 460, 801

Fryer, C. L., Woosley, S. E., \& Hartmann, D. H. 1999, ApJ, 526, 152

Gehrels, N., Sarazin, C. L., O'Brien, P. T., et al. 2005, Nature, 437, 851

Giacconi, R., \& Ruffini, R. 1978, Physics and astrophysics of neutron stars and black holes (Amsterdam: North Holland Publishing)

Gursky, H., \& Ruffini, R. 1975, Neutron stars, black holes and binary X-ray sources; Proceedings of the Annual Meeting, San Francisco, Calif., February 28, 1974, (Dordrecht: D. Reidel Publishing), Astrophys. Space Sci. Lib. 48

Izzo, L., Bernardini, M. G., Bianco, C. L., et al. 2010, JKPS, 57-3, 551

Izzo, L., Rueda, J. A., \& Ruffini, R. 2012a, A\&A, 548, L5

Izzo, L., Ruffini, R., Penacchioni, A. V., et al. 2012b, A\&A, 543, A10

Kann, D. A., Klose, S., Zhang, B., et al. 2011, ApJ, 734, 96

Kouveliotou, C., Granot, J., Racusin, J. L., et al. 2013, ApJ, 779, L1

Krimm, H. A., Beardmore, A. P., Evans, P. A., et al. 2009, GRB Coordinates Network, 9198, 1

Margutti, R., Zaninoni, E., Bernardini, M. G., et al. 2013, MNRAS, 428, 729 
R. Ruffini et al.: IGC at high redshift

Meegan, C., Lichti, G., Bhat, P. N., et al. 2009, ApJ, 702, 791

Melandri, A., Pian, E., D’Elia, V., et al. 2014, A\&A, 567, A29

Meszaros, P. 2006, Rep. Prog. Phys., 69, 2259

Meszaros, P., \& Rees, M. J. 2000, ApJ, 530, 292

Metzger, B. D., Martínez-Pinedo, G., Darbha, S., et al. 2010, MNRAS, 406, 2650

Nousek, J. A., Kouveliotou, C., Grupe, D., et al. 2006, ApJ, 642, 389

Page, K. L., Starling, R. L. C., Fitzpatrick, G., et al. 2011, MNRAS, 416, 2078

Patricelli, B., Bernardini, M. G., Bianco, C. L., et al. 2012, ApJ, 756, 16

Penacchioni, A. V., Ruffini, R., Izzo, L., et al. 2012, A\&A, 538, A58

Penacchioni, A. V., Ruffini, R., Bianco, C. L., et al. 2013, A\&A, 551, A133

Pisani, G. B., Izzo, L., Ruffini, R., et al. 2013, A\&A, 552, L5

Rueda, J. A., \& Ruffini, R. 2012, ApJ, 758, L7

Ruffini, R. 2011, Int. J. Mod. Phys. D, 20, 1797

Ruffini, R. 2013, 27th Texas Symposium on Relativistic Astrophysics, Dallas, TX (USA), December 8-13

Ruffini, R., Izzo, L., Muccino, M., et al. 2013, [arXiv: 1311.7432]
Ruffini, R., Muccino, M., Bianco, C. L., et al. 2014a, A\&A, 565, L10 Ruffini, R., Wang, Y., Kovacevic, M., et al. 2014b, ApJ, submitted [arXiv: 1405. 5723]

Salvaterra, R., Della Valle, M., Campana, S., et al. 2009, Nature, 461, 1258

Salvaterra, R., Campana, S., Vergani, S. D., et al. 2012, ApJ, 749, 68

Starling, R. L. C., Page, K. L., Pe'Er, A., Beardmore, A. P., \& Osborne, J. P. 2012, MNRAS, 427, 2950

Stratta, G., \& Perri, M. 2009, GRB Coordinates Network, 9212, 1

Tanvir, N. R., Fox, D. B., Levan, A. J., et al. 2009, Nature, 461, 1254

von Kienlin, A. 2009a, GRB Coordinates Network, 9229, 1

von Kienlin, A. 2009b, GRB Coordinates Network, 9251,

Woosley, S. E. 1993, ApJ, 405, 273

Woosley, S. E., Heger, A., \& Weaver, T. A. 2002, Rev. Mod. Phys., 74, 1015

Zel'dovich, Y. B., Ivanova, L. N., \& Nadezhin, D. K. 1972, Sov. Astron., 16, 209

Zhang, B., Fan, Y. Z., Dyks, J., et al. 2006, ApJ, 642, 354 